Research article

Open Access

\title{
Formulation and Evaluation of Diltiazem Hydrochloride Gels for the Treatment of Anal Fissures
} \author{
Sanju Dhawan * ${ }^{1}$, Bikash MedhI ${ }^{2}$, Sunny ChOPRA ${ }^{1}$ \\ ${ }^{1}$ University Institute of Pharmaceutical Sciences, Center for Advanced Studies, Panjab University, \\ Chandigarh, India. \\ ${ }^{2}$ Department of Pharmacology, Post Graduate Institute of Medical Education and Research, Chandigarh, \\ India. \\ * Corresponding author. E-mail: sanjudhawan@rediffmail.com (S. Dhawan)
}

Sci Pharm. 2009; 77: 465-482

doi:10.3797/scipharm.0903-10

Published: $\quad$ May $5^{\text {th }} 2009$

Accepted: $\quad$ May $5^{\text {th }} 2009$

Received: $\quad$ March $14^{\text {th }} 2009$

This article is available from: http://dx.doi.org/10.3797/scipharm.0903-10

(C) Dhawan et al.; licensee Österreichische Apotheker-Verlagsgesellschaft m. b. H., Vienna, Austria.

This is an Open Access article distributed under the terms of the Creative Commons Attribution License (http://creativecommons.org/licenses/by/3.0/), which permits unrestricted use, distribution, and reproduction in any medium, provided the original work is properly cited.

\begin{abstract}
The treatment for chronic anal fissure (CAF) has undergone a transformation in recent years from surgical to medical. Both the approaches share the common goal of reducing the spasm of anal sphincter. Though surgical treatment has a high success rate but it can permanently impair fecal continence in large number of patients. Smooth muscle relaxation seems to be a novel way by which more than $60 \%$ of the patients can be cured with the topical use of the agents. In the present investigation, diltiazem hydrochloride gels were prepared using FDA recommended polymers [hydroxypropylmethyl cellulose (HPMC), methylcellulose (MC) and polyethylene oxide (PEO grade 301 and 303)] for topical application in CAF. Increasing the concentration of the polymer significantly increased the consistency of the gels. All the formulations exhibited pseudoplastic flow with no thixotropy. The values of flow index (n) were found to be less than one for all the gels confirming the shear thinning behaviour of all the gels. HPMC and MC gels were found to be stable at accelerated stability conditions while the bioadhesion of PEO gels was highest. Even after exposure to heat and humidity, no significant change was observed in the content uniformity, $\mathrm{pH}$, clarity, texture profile analysis and rheological properties of the HPMC and MC gels. The rheograms and various power law equation parameters of these gels were found comparable at various time points in the accelerated stability study. However, PEO gels failed in accelerated stability studies at one month sample. When four selected gel formulations (HPMCL4, MCL3, P1BL3 and P3BL3.5) were applied topically by six patients each,
\end{abstract}


Gastrointestinal Quality of Life Index (GIQLI) score of each patient was improved at the end of 8 weeks. No adverse effects were reported by any of the patients. Hence $2 \%$ DTZ gel was found to be effective in the treatment of anal fissures.

\section{Keywords}

Bioadhesion - Chronic anal fissure - Diltiazem hydrochloride - Gastrointestinal quality of life index $•$ Rheology

\section{Introduction}

According to Antropoli et al., various pathologies of anal canal are extremely common $[1,2]$. About $30-40 \%$ of the population suffers from proctologic pathologies at least once in their lives. Anal fissure (AF) is present in about $10-15 \%$ of proctological patients [3]. AF can be defined as a tear or split in the distal anal canal, which if not treated appropriately at an early stage causes considerable anal pain during defecations $[4,5]$. It is associated with spasm of the internal anal sphincter and a reduction in mucosal blood flow with delayed or nonhealing of the ulcer $[6,7]$. The primary cause of chronic anal fissure (CAF) is increased resting anal pressure. Other most frequent causes are infection, essentially sexually transmitted diseases, and tumor, mainly anal epidermoid cancer. Most AFs heal spontaneously with conservative treatment, viz., stool softener and diet modification. Such AFs are termed as acute but a proportion of them persists for a longer period and is known as chronic. Chronicity is defined by both chronology and morphology. Most surgeons consider the persistence for 6 weeks as a reasonable point when an AF, unlikely to heal with conservative treatment, may be considered chronic. Morphologically, the presence of visible transverse internal anal sphincters fibers at the base of a fissure typifies chronicity. Associated features include indurated edges, a sentinel pile, and a hypertropical anal papilla. An acute fissure looks like a fresh tear in the skin, while in a CAF the walls of the tear become thickened [8].

The treatment for CAF is based on reducing the spasm of the internal anal sphincter, either by dilating the anal canal or sphincterotomy [9]. Lateral Internal Sphincterotomy (LIS) is a surgical technique to cure AF. It has been favored by most of the surgeons, because it offers long-lasting relief in sphincter spasm [10,11]. Traditionally, LIS is considered as the gold standard treatment for chronic fissures, but it permanently weakens the internal sphincter and may lead to anal deformity and incontinence in $8-30 \%$ of patients $[12,13]$. Therefore, recently, nonsurgical treatment modalities have been developed. Smooth muscle relaxation is an effective treatment for CAF and has advantages over surgical treatment in avoiding long term complications. Additionally, it does not require hospitalization [14]. Topical application of various agents viz., glyceryl trinitrate $(0.2 \%)$, diltiazem $(2 \%)$, nifedipine $(0.2 \%)$, L-arginine $(400 \mathrm{mg})$, minoxidil $(0.5 \%)$, lignocaine $(5 \%)$, isosorbides $(0.2 \%)$ is being exploited for the treatment of AF. Diltiazem $(60 \mathrm{mg})$ and lacidipine $(6 \mathrm{mg})$ have also been tried orally. In addition to these approaches, injections of botulinum toxin $(30 \mathrm{U}-50 \mathrm{U})$ and gonyautoxin $(100 \mathrm{U})$ have also been reported [15]. All of these studies have shown that surgery can be avoided in 33 to $98 \%$ of patients by using smooth muscle relaxation. A variety of agents employed for relaxation of 
smooth muscles have been reviewed [8]. The reviewers proposed an algorithm for the treatment of anal fissure

As postulated by Dhawan and Chopra, diltiazem hydrochloride ( $2 \%$ topical) is the first line treatment in case of CAF, it was decided to formulate this active ingredient in the form of gels for topical application to CAF. In the present investigation, the gels were subjected to in vitro evaluation studies viz., rheology, Texture Profile Analysis (TPA), $\mathrm{pH}$, bioadhesion, in vitro permeation studies and cloth staining. In addition, clinical studies in healthy volunteers and patients were also conducted in collaboration with PGIMER, Chandigarh.

\section{Materials}

Diltiazem hydrochloride (DTZ) was obtained from Cipla Ltd., Mumbai, India. Hydroxypropyl Methylcellulose (HPMC) and Methyl Cellulose (MC) were procured from Overseas Healthcare Pvt. Ltd., Phillaur, India and E. Merck Ltd., Mumbai, India respectively. Polyethylene oxide (PEO) (WSR 301 and 303) were procured from Union Carbide Corporation, USA. All other reagents were of analytical grade.

\section{Methods}

\section{Analytical Method Validation of DTZ}

The analytical method was validated for linearity, accuracy and precision. The spectrophotometric standard plots of DTZ were prepared in water, buffers of $\mathrm{pH}$ 2.8, 3.7, 4.7, 5.0, 6.0 and phosphate buffer saline. Linearity of the method was validated by measuring absorbance at different concentration range and plotting calibration curves. Accuracy was determined by spiking polymeric solutions with six different concentration of DTZ and comparing the absorbance with that of the solution prepared in the same way using water/buffer but without polymer. The average accuracy and standard deviation were calculated. Precision was validated by measuring absorbance at six concentrations. Precision was expressed by the standard deviation (SD) and relative standard deviation (RSD) of measured response. The intermediate precision was evaluated by determining the absorbance of the different concentrations of DTZ (stored in refrigerator) on different days.

\section{Partition Coefficient Determination}

Partition Coefficient (PC) was determined in n-octanol and buffers of different $\mathrm{pH}$. Two solvents were mutually saturated before use, by shaking them together in equal volumes, for $24 \mathrm{~h}$ at $37{ }^{\circ} \mathrm{C}$ or ambient temperature. After centrifugation at $2000 \mathrm{rpm}$ for $20 \mathrm{~min}$, the octanol layer was pipetted out. A stock solution of drug was prepared in respective buffer. The drug stock solution $(2 \mathrm{~mL})$ and saturated octanol $(13 \mathrm{~mL})$ were taken in a $25 \mathrm{~mL}$ conical flask that was capped tightly and shaken on a platform shaker for $24 \mathrm{~h}$ at $37{ }^{\circ} \mathrm{C}$ or ambient temperature. The two liquids were then separated and analyzed for DTZ content using the standard plot in distilled water/buffer. The partition/distribution coefficient was calculated according to the equation 1.

Eq. 1. $\quad P C=\frac{C_{0}}{C_{w}} \times \frac{V_{w}}{V_{0}}$ 
Where $C_{o}$ is the concentration in oil phase, $C_{w}$ is the concentration in aqueous phase, $V_{w}$ is the volume of aqueous phase and $V_{0}$ is the volume of oil phase

\section{Preparation of gels}

MC gels were prepared with the various concentrations of $\mathrm{MC}(1 \%, 2 \%$ and $3 \%)$ as reported by Attia et al., [16]. Two grams of DTZ was dissolved in $10 \mathrm{~mL}$ of water. Required amount of MC and methyl paraben sodium $(120 \mathrm{mg})$ were dissolved separately in warm water and stirred with the help of glass rod for $5 \mathrm{~min}$ and then mixed. DTZ solution was added to MC solution and final weight was made up to $100 \mathrm{gm}$ with water. The gel was stirred with a mechanical stirrer at a speed of $100 \mathrm{rpm}$ for $15 \mathrm{~min}$. Unloaded gels were prepared in the same way except DTZ was not added. The gel was kept in a vacuum oven at room temperature to remove entrapped air. The gel was filled in the tubes and stored at 8-15 ${ }^{\circ} \mathrm{C}$. HPMC gels were also prepared in the same manner as MC gels. PEO gels were prepared by dissolving $2 \mathrm{gm}$ of DTZ and methyl paraben sodium $(0.2 \% \mathrm{w} / \mathrm{w})$ in minimum amount of water, then required amount of PEO was added and weight was made up with water. This was then stirred with a mechanical stirrer at a speed of $500 \mathrm{rpm}$ for $30 \mathrm{~min}$ [17]. The gel was then kept in a vacuum oven at room temperature to remove any entrapped air. PEO gels were also prepared with acetate buffer $(\mathrm{pH} 5)$ in exactly the same way as mentioned above except that water was replaced with buffer. Gels with two grades of PEO (WSR 301 and WSR 303) were prepared and subjected to evaluation. Table 1 describes the composition of various gels prepared during the study.

\section{Evaluation of gels}

\section{Visual appearance}

The prepared gels were visually inspected for clarity, color and transparency. The prepared gels were also evaluated for the presence of any particles. Smears of gels were prepared on glass slide and observed under the microscope for the presence of any particle or grittiness.

pH of the gels

The $\mathrm{pH}$ of gel was determined after diluting and dispersing it in distilled water $(10 \% \mathrm{w} / \mathrm{v})$. All the measurements were made in triplicate and mean calculated.

\section{Drug Content Estimation}

Exactly $0.1 \mathrm{~g}$ gel was completely dispersed in distilled water to make final volume $100 \mathrm{~mL}$ $(0.1 \% \mathrm{w} / \mathrm{v})$ by subjecting it to stirring $(400 \mathrm{rpm})$ for $5 \mathrm{~min}$. The dispersion was than filtered to remove the undissolved residue. Exactly $1 \mathrm{~mL}$ of the filtrate was diluted to $5 \mathrm{~mL}$ and absorbance was measured at $236 \mathrm{~nm}$. An unloaded gel was also subjected to a similar determination to observe the effect of excipients on the absorbance. Using the standard curve of DTZ in distilled water, the drug content in gel was finally estimated. Three batches of each polymer concentration were subjected to this determination. 
Tab. 1. Codes and composition of gels prepared in the investigation

\begin{tabular}{llcl}
\hline Code & Polymer & $\begin{array}{c}\text { Polymer } \\
\text { Concentration } \\
\text { (\% w/w) }\end{array}$ & Solvent \\
\hline HPMCL1 & HPMC & 1 & Water \\
HPMCL2 & HPMC & 2 & Water \\
HPMCL3 & HPMC & 3 & Water \\
HPMCL4 & HPMC & 4 & Water \\
HPMCU4 & HPMC & 4 & Water \\
MCL1 & MC & 1 & Water \\
MCL2 & MC & 2 & Water \\
MCL3 & MC & 3 & Water \\
MCU3 & MC & 3 & Water \\
P1WU1 & PEO 301 & 1 & Water \\
P1WL1 & PEO 301 & 1 & Water \\
P1BL1 & PEO 301 & 1 & Acetate Buffer (pH-5) \\
P1BL2 & PEO 301 & 2 & Acetate Buffer (pH-5) \\
P1BL3 & PEO 301 & 3 & Acetate Buffer (pH-5) \\
P1BU3 & PEO 301 & 3 & Acetate Buffer (pH-5) \\
P3WU1 & PEO 303 & 1 & Water \\
P3WL1 & PEO 303 & 1 & Water \\
P3BL1 & PEO 303 & 1 & Acetate Buffer (pH-5) \\
P3BL2 & PEO 303 & 2 & Acetate Buffer (pH-5) \\
P3BL3 & PEO 303 & 3 & Acetate Buffer (pH-5) \\
P3BL3.5 & PEO 303 & 3.5 & Acetate Buffer (pH-5) \\
\hline Formulations with alphabet L in their code contain 2\% w/v DTZ \\
Formulations with alphabet U in their code contain no drug
\end{tabular}

\section{Rheology of gels}

Rheological studies of different formulations were performed at $20.4 \pm 0.1 \mathrm{C}$ using a Haake (RT-20) rheometer. The diameter of plate was $20.05 \mathrm{~mm}$ and cone angle was $1^{\circ}$. The interplate diameter was $0.052 \mathrm{~mm}$ and truncation was 0.05 '. Rheograms were produced in duplicate by gradually increasing the shear stress. The shear stress value was increased automatically by the instrument to a maximum value of $500 \mathrm{~Pa}$ over a period of $30 \mathrm{~s}$. The relationship between shear stress and shear rate of each formulation was determined using Power law described in equation 2.

Eq. 2. $\tau=k \gamma^{n}$

All rheological analyses were performed in duplicate. Rheograms of optimized formulations HPMCL4, MCL3, P1BL3 and P3BL3.5 were statistically evaluated by three way analysis of variance (ANOVA).

Texture Profile Analysis (TPA) 
The color and texture (relating to smoothness and uniformity) of the gel were evaluated manually. Sensory properties included measures such as consistency, firmness, cohesiveness (attractive forces within the formulation) and work of adhesion (attraction between the formulation and the substrate). The textural/mechanical properties of the gel formulation were measured using a TA-XT2 texture analyzer (Stable Microsystem, Surrey UK) in TPA mode. Tests were carried out in Mc Cartney bottles (height $47 \mathrm{~mm}$; diameter $30 \mathrm{~mm}$; sample height in the container $27 \mathrm{~mm}$ ), immediately after removal from storage at $25^{\circ} \mathrm{C}$. A defined mass of each formulation $(20 \mathrm{gm})$ was transferred into Mc Cartney bottles and filled to a fixed height avoiding the introduction of air bubbles. After this, the temperature of each sample was allowed to equilibrate to $20^{\circ} \mathrm{C} \pm 1{ }^{\circ} \mathrm{C}$ by stage in an oven for $24 \mathrm{~h}$. The extrusion disc was positioned centrally over the sample container. It was made sure that the testing surface was as flat as possible to avoid early triggering of the test. The probe was programmed to descend into the sample at a speed of $2 \mathrm{~mm} \mathrm{~s}^{-1}$ to a depth of $30 \mathrm{~mm}$ and then ascend back at the same speed to its original position. The force encountered by the probe to break away from the gel when starting to ascend (the point of maximum force) was measured. Care was taken so that the extrusion disc does not come into contact (or indeed approach very close) to either the walls or base of the container during testing which could produce an erroneous result.

\section{Bioadhesion measurement}

Bioadhesion measurement was based on the principle of recording force required to break the adhesive bond between a model membrane and the test formulation. Bioadhesive strength of the gel was measured using a calibrated texture analyzer equipped with a 5 $\mathrm{kg}$ load cell and modified probes. The probes of equipment were modified suitably so as to hold cellophane membrane, hydrated with PBS 7.4. Data was acquired at a rate of 50 points $\mathrm{sec}^{-1}$, using fully integrated data acquisition and analysis software, i.e.,. Texture Expert for Windows Version 1.2.2. Single unit dose of gel $(0.5 \mathrm{~g}$ gel dispersed in $3 \mathrm{~mL}$ normal saline) was applied in between layers of cellophane sheet, on an area of $464 \mathrm{~mm}^{2}$. Membranes were kept in contact with the test sample for a period of $5 \mathrm{~min}$, under a constant force of $0.25 \mathrm{~N}$, in order to establish a proper contact between the membrane and sample, and to allow the formation of an adhesive bond. Force required to separate the two membranes (with gel in between) was measured by the upper support of texture analyzer moving at a rate of $0.1 \mathrm{~mm} \mathrm{sec}{ }^{-1}$. Maximum force required to break the bond was measured as bioadhesive strength and area under curve was measured as work of adhesion. Formulations analyzed include HPMCL4, MCL3, P1BL3, P3BL3.5. For all the formulations, mean bioadhesive strength and work of adhesion were determined in replicates $(n=3)$ and the mean values determined.

\section{In vitro permeation studies}

In vitro diffusion studies were carried out for all the gels in triplicate, using Franz Diffusion cell [18]. The receptor compartment was filled with water. The two compartments (donor and receptor) were also glued to each other with the acrylate adhesive. The gel was placed on the membrane in the donor compartment. An aliquot sample was periodically withdrawn at suitable time intervals from the receptor compartment and equal volume replaced. The samples were analyzed spectrophotometrically at $237 \mathrm{~nm}$. The raw data obtained from diffusion release studies was analyzed using Zorel software. The software has the inbuilt provision for applying the correction factor for volume and drug losses 
during sampling and calculating the values of amount and percent release at various time intervals [19].

\section{Cloth staining}

This study was undertaken to assess the fabric staining property of the gels. Fabrics of different fiber blends ranging from $100 \%$ cotton to $100 \%$ polyester were procured and stained with $0.5 \mathrm{gm}$ gels of DTZ and observed for staining after washing.

All sample clothes were cut into $6 \mathrm{sq}$ inch squares and DTZ gels $(0.5 \mathrm{~g})$ were applied in the center of each cloth. The stains were air-dried and the clothes left at $50{ }^{\circ} \mathrm{C}$ for $48 \mathrm{~h}$ to accelerate the drying. After leaving them at room temperature for 5 more days (total 1 week), they were washed with standard detergent Surf ${ }^{\circledR}$ and water. After air-drying the staining on the sample clothes was recorded.

\section{Evaluation of containers for gels}

The collapsible aluminium tubes were tested for freedom from leakage by placing filled tubes in a horizontal position on a sheet of absorbent blotting paper in an oven maintained at $60 \pm 3{ }^{\circ} \mathrm{C}$ for $8 \mathrm{~h}$ and observing for leakage [20]. The tubes were tested for freedom from metal particles by observing the smears of gels under light microscope.

\section{Stability testing}

The gels were visually inspected everyday for seven weeks for any change in physical appearance of gels i.e., color, turbidity, odor etc. All the gels were subjected to stability studies by keeping them at accelerated conditions of temperature and humidity $\left(40 \pm 2{ }^{\circ} \mathrm{C}\right.$, $75 \pm 5 \% \mathrm{RH}$ ) for a period of 6 months. Zero time samples were used as controls. Samples withdrawn at predetermined intervals $(0,1,3$ and 6 months) were analyzed for various performance parameters i.e., $\mathrm{pH}$, rheology, microbial growth and drug content. Gels that failed in accelerated stability studies were stored at a temperature of $8-15{ }^{\circ} \mathrm{C}$ (in a refrigerator). Samples from these gels were withdrawn at predetermined intervals $(0,1,3$ and 6 months) and analyzed for abovementioned performance parameters. These gels were also subjected to freeze-thaw study. In this study, samples were kept in refrigerator for 2 days followed by room temperature for two days. Five cycles were carried out and at the end, performance parameters were determined.

\section{Clinical studies}

PGIMER ethics committee approval was obtained for the study. The approval from Drugs Controller General of India (vide letter no 4-82/04-DC) was also sought. Twenty four patients who fulfilled the inclusion criteria were recruited for the study. Patients of either sex (> 18 years of age) with diagnosis of CAF were included. Exclusion criteria were presumed or confirmed pregnancy, allergy to DTZ, associated condition warranting surgery e.g. abscess, fistula and anal cancer, concomitant first degree to third degree hemorrhoids, patients with other associated disease condition such as Crohn's disease, HIV infection (suspected), fistula in ano, anal abscess or other medical unstable conditions and patients with previous surgery in anal canal. The written consent was obtained from each patient. At the time of enrollment, the patients were subjected to general examination, systemic examination (CVS (ECG), Respiratory, CNS, GIT), local examination, and biochemical investigations viz., Hb, TLC, DLC, LFT (SGOT, SGPT, 
alkaline phosphatase, bilirubin, proteins), RFT (serum urea, serum creatinine). The patients were asked to answer the questionnaire Gastrointestinal Quality of Life Index (GIQLI) and a baseline score was calculated on the first visit [21]. Similarly the scores were calculated after reviewing the patients at 8 weeks of the study. The gels were also evaluated on the basis of compliance score based on a questionnaire. Depending upon the answers given by the patients on the first visit and after the treatment, total scores were calculated. The scores obtained before and after the completion of treatment were subjected to paired t test using Sigmastat version 2.0. The compliance scores of different gels were compared on the basis of one way ANOVA followed by Dunnet's test.

\section{Results and Discussion}

\section{Validation of analytical method}

\section{Linearity}

The linearity of a method is a measure of how well a calibration plot of response vs. concentration approximates a straight line, or how well the data fit to the linear equation described in equation 3.

\section{Eq. 3.}

$$
y=m x+c
$$

Where $y$ is the response, $x$ is the concentration, $m$ is the slope and $c$ is the intercept of a best fit line to the data. Ideally a linear relationship is preferred because it is more precise, easier for calculations, and can be confined with fewer standards. Also, UV detector response for a dilute sample is expected to follow Beer's law, and be linear. A linear relationship was observed between the absorbance and concentration in 2-20 $\mathrm{\mu g}$ range as evident from $r^{2}$ values $(>0.9980)$.

\section{Accuracy}

Accuracy is defined as the closeness of the measured value to the true value. The true value can be obtained by direct comparison to a standard or by analyte recovery or by method of standard addition. Since DTZ was to be quantitated in the gels, accuracy was calculated by comparing the measured value (obtained by dilution of the gel) with standard value (in gel solvent buffer/ water). The average accuracy was found to be $99.71 \%$, 99.95\%, $99.78 \%$ and $99.8 \%$ for HPMC, MC, PEO (301) and PEO (303) respectively. The respective $\mathrm{RSD}$ values for the above-mentioned gels were found to be $1.029,1.041,1.012$ and 1.072 indicating very good accuracy.

\section{Precision}

Precision was measured by the sequential, repetitive measuring of absorbance of the same homogenous sample (typically 5 to 10 times), followed by the averaging of the absorbance values and determination of the RSD of all concentrations. RSD values obtained for the data indicated a high degree of repeatability.

\section{Partition coefficient}

The log $P$ values of DTZ with octanol/water and octanol/buffer system were found to be about -2 , indicative of highly hydrophilic nature of DTZ. In addition, $\log P$ values decreased with increase in $\mathrm{pH}$. This behavior is ascribed to decrease in solubility of the 
drug with increase in $\mathrm{pH}$. The log $\mathrm{P}$ values were found to be in accordance with the reports in the literature [21].

\section{Evaluation of gels}

\section{Visual appearance}

All the four gels containing DTZ were found to be transparent and uniform in consistency, except the gels of PEO prepared in water.

\section{Presence of particulate matter (grittiness)}

All the formulations were evaluated microscopically for the presence of particulate matter. No appreciable particulate was seen under microscope. Hence, the gel formulations fulfilled the requirement of freedom from particulate matter and from grittiness, as desired for any topical preparation.

\section{pH determination}

The $\mathrm{pH}$ of each gel after dispersion in distilled water was noted and the results were taken as a mean of three determinations (Table 2). When MC and HPMC wee used for the preparation of gels, clear gels were obtained in distilled water. However, when PEO gels were prepared in water, it led to precipitation of DTZ (Figure 1B). The needle shaped crystals of DTZ were observed. The identity of the crystals was confirmed by increasing the $\mathrm{pH}$ of DTZ solution in water (DTZ gets precipitated at $\mathrm{pH}>7$ ) and observing the solution under optical microscope (Figure 1C). The gels prepared using buffers of different $\mathrm{pH}$ were observed under microscope to visualize any precipitation of the active ingredient at different $\mathrm{pH}$ values (Figure 1).

Tab. 2. The values of $\mathrm{pH}$ for various DTZ gels

\begin{tabular}{lrlr}
\hline \multicolumn{1}{c}{ GEL CODE } & $\mathbf{p H}$ & GEL CODE & $\mathbf{p H}$ \\
\hline HPMCL1 & 5.19 & P1WL1 & 6.27 \\
HPMCL2 & 5.21 & P1BL1 & 5.25 \\
HPMCL3 & 5.22 & P1BL2 & 5.29 \\
HPMCL4 & 5.24 & P1BL3 & 5.33 \\
HPMCU4 & 5.31 & P1BU3 & 5.38 \\
MCL1 & 5.11 & P3WU1 & 6.62 \\
MCL2 & 5.13 & P3WL1 & 6.30 \\
MCL3 & 5.14 & P3BL1 & 5.31 \\
MCU3 & 5.19 & P3BL2 & 5.34 \\
P1WU1 & 6.54 & P3BL3.5 & 5.35
\end{tabular}

\section{Drug content estimation}

DTZ content of all the gels was estimated by withdrawing samples at random from three different sampling points in a single batch of the gel. Three batches were estimated in similar manner. Estimations were made spectrophotometrically, after dispersion of the gel in distilled water. The content of DTZ in all the gels was found to be within limits (>97\%). Samples within a batch were uniform as evident from the low standard deviation value (<4.5\%). 


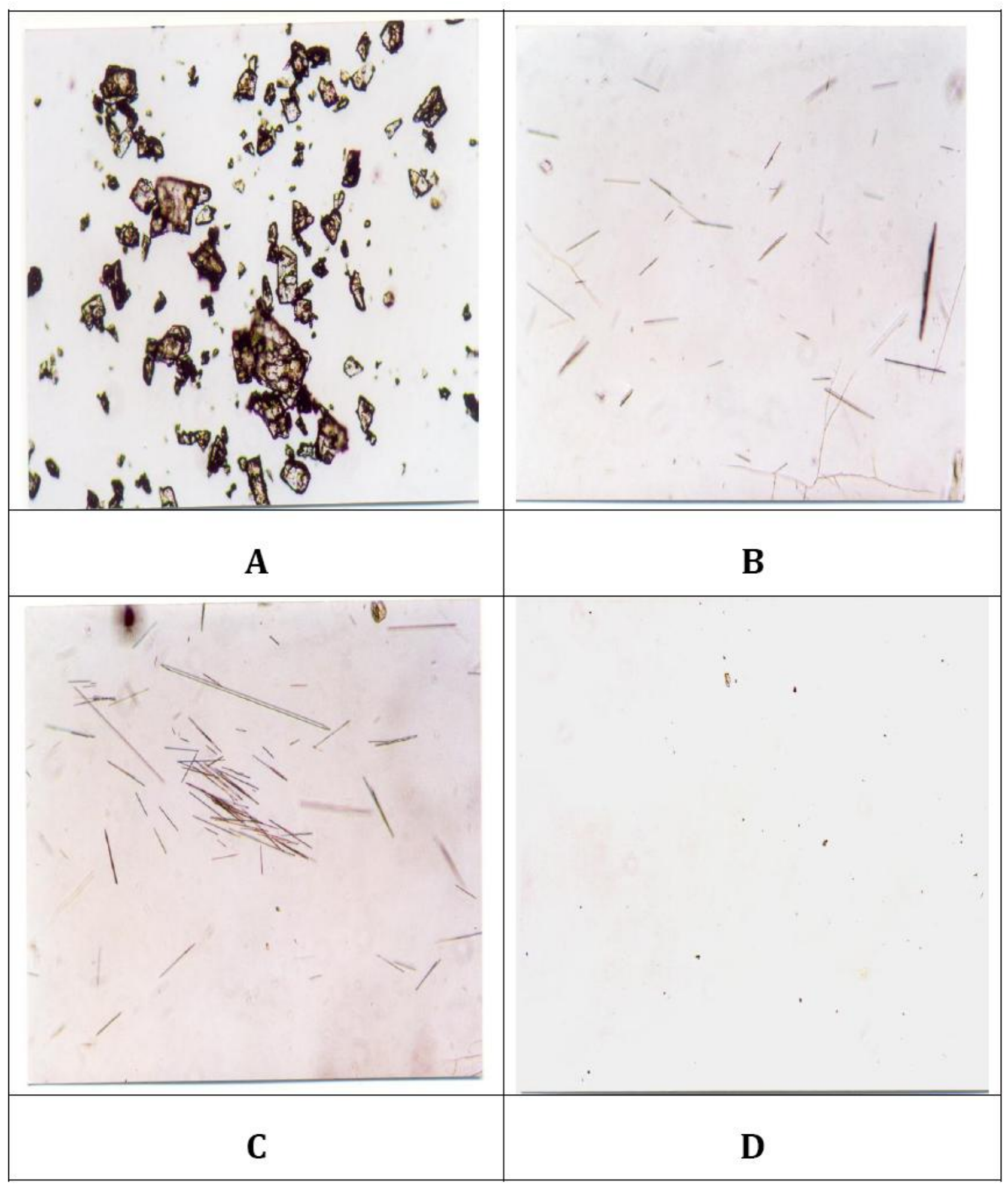

Fig. 1. (A) Crystals of pure DTZ; (B) Crystals of DTZ observed in Gels of PEO prepared using water; (C) Crystals of DTZ due to forced precipitation at high $\mathrm{pH}$; (D) PEO gel formed in acetate buffer $(\mathrm{pH} 5)$ showing no precipitation. 


\section{Rheology of gels}

Increasing the concentration of the polymer significantly increased the consistency. Increased consistency was ascribed to enhanced polymeric entanglements, thereby increasing the resistance to deformation. All the formulations exhibited pseudoplastic flow; however no evidence of thixotropy was observed (Figure 2 and 3). Application of the power law model to the rheological properties of each formulation enabled the calculation of the consistency $(k)$ and flow index $(n)$. The values of flow index $(n)$ were found to be less then one for all the gels confirming the shear thinning behavior of all the gels. The same is also confirmed from plots of viscosity vs. shear rate indicating that the viscosity of the system decreased with increase in shear rate. The gels did not break even at shear rate of 500 indicating good gel strength. The values of flow index for HPMCL4 was highest (0.419) followed by MCL3 (0.380), P3BL3.5 (0.357) and P1BL3 (0.329). High flow index reflects the mobility of the gel from the container. The values of consistency index for HPMCL4 and MCL3 were found to be higher then P1BL3 and P3BL3.5 $(P<0.01)$.

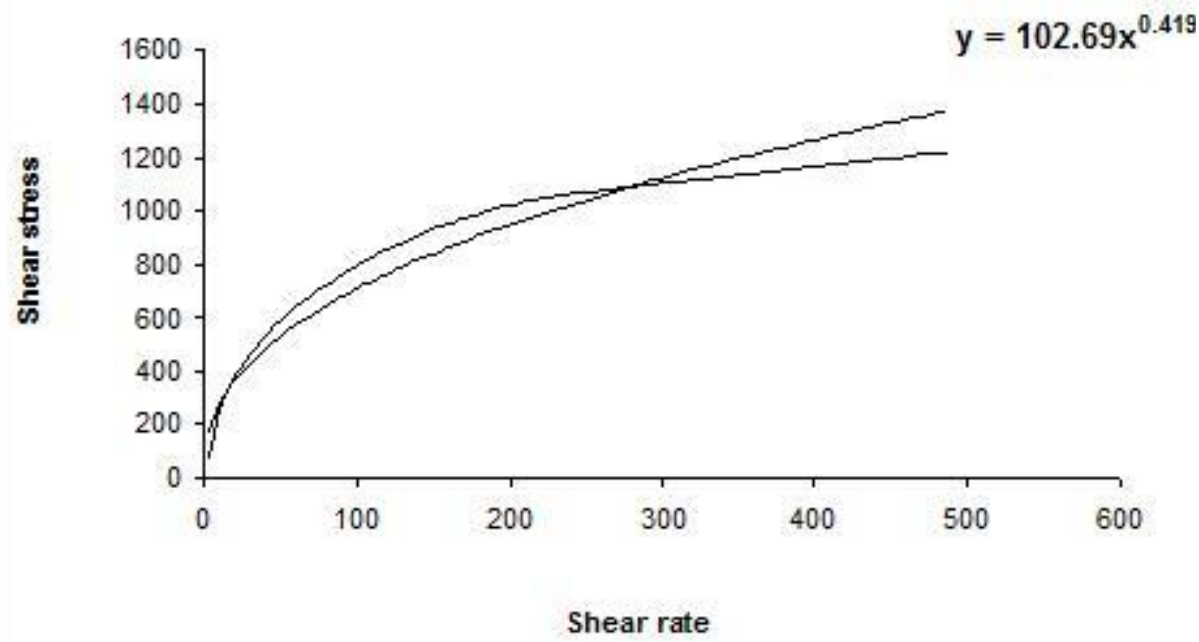

Fig. 2. Representative rheogram of HPMCL4 depicting power law

\section{Physical and sensory properties}

Formulations, which are designed for topical application/body cavities, must exhibit acceptable mechanical characteristics e.g., ease of application. One method by which the mechanical properties of polymeric systems may be conveniently determined is texture profile analysis (TPA). In this, an analytical probe is twice depressed into the sample at a defined rate to a desired depth, allowing a predefined necessary period between the end of the first and the beginning of second compression [22]. The peak or maximum force is taken as a measurement of firmness; the higher the value, the more firm is the sample (Figure 4). The area of the curve up to this point is taken as a measurement of consistency. The higher the value the thicker is the consistency of the sample. The negative region of the graph, produced on probe return, is as a result of the weight of sample which is lifted primarily on the upper surface of the disc on return, i.e., due to back extrusion and hence gives again an indication of consistency/resistance to flow off the disc. The maximum negative force is taken as an indication of the stickiness/cohesiveness of the sample. The more negative the value the more 'sticky' or 'cohesive' is the sample. 
Area of the negative region of the curve is often referred to as work of adhesion/viscosity. Higher is the value, more resistant to withdrawal is the sample, which is an indication of resistance to flow/viscosity of the sample.

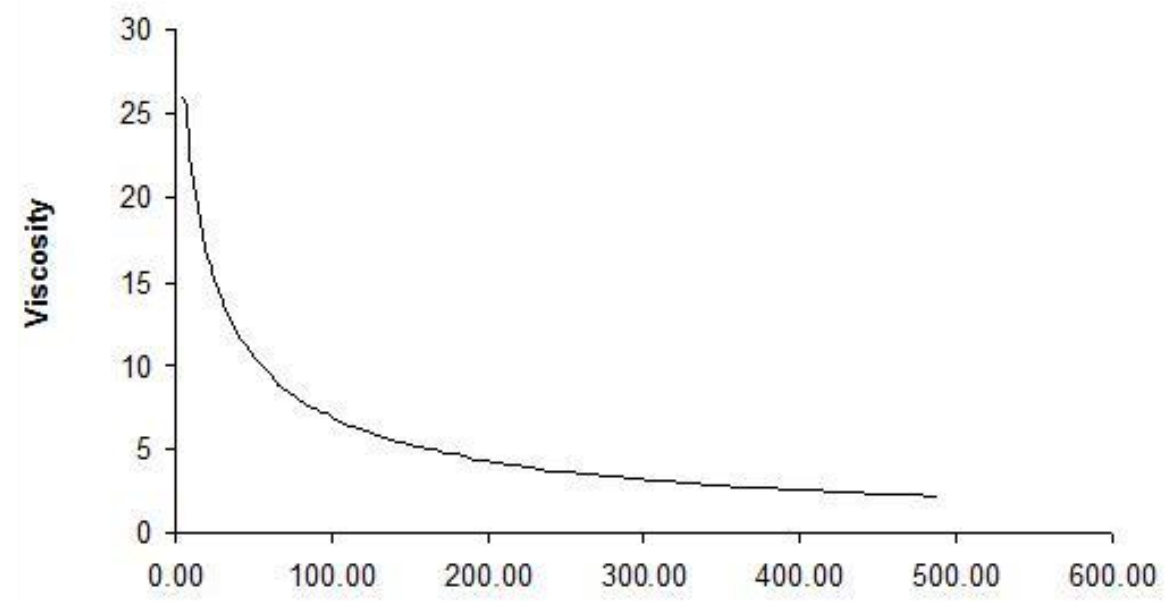

Shear rate

Fig. 3. Representative plot of Viscosity vs. Shear Rate for HPMCL4

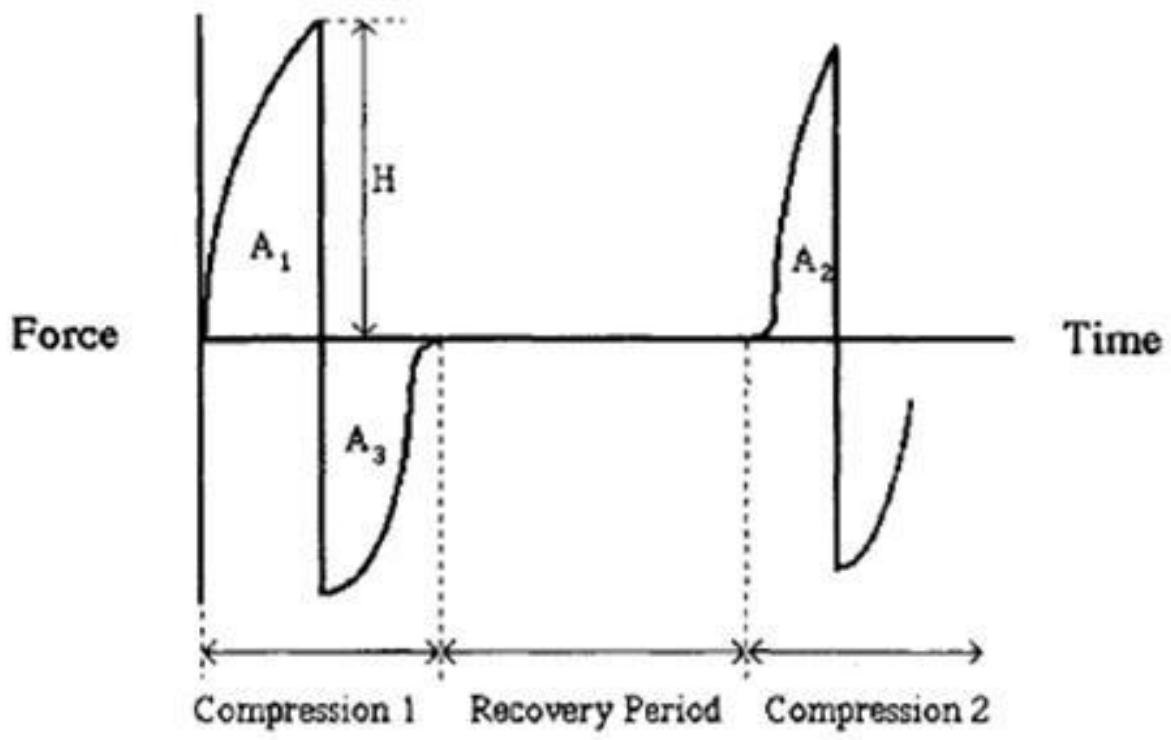

Fig. 4. Graphical output from texture profile analyzer

In Figure $4, \mathrm{H}$ is the firmness/hardness, $A_{1}$ is compressibility, $A_{3}$ is adhesiveness and $A_{2} /$ $A_{1}$ is cohesiveness. Table 3 compares the various sensory properties of the gels. The table indicates that HPMCL4 is having maximum firmness, work of shear, stickiness and work of adhesion whereas P3BL3.5 is having least firmness, work of shear and stickiness and work of adhesion. 


\section{Bioadhesion measurement}

Formulations that have been designed for topical use should exhibit adhesion to mucosal surfaces, as this will improve the clinical efficacy. The method to measure bioadhesion was based on the principle of measuring the force required to break the adhesive bond between cellophane membrane and the test formulation using texture analyzer. The test formulation was sandwiched between two cellophane membranes fixed on flexible supports in the modified probes of texture analyzer for a sufficient period of time. After the adhesive bond had formed, the force required to separate the bond was measured as the bioadhesive strength. Figure 5 represents the comparative bioadhesive strengths of gels.

Tab. 3. Comparison of sensory properties of optimized gels

\begin{tabular}{lcccc}
\hline \multicolumn{1}{c}{ Property } & HPMCL4 & MCL3 & P1BL3 & P3BL3.5 \\
\hline Firmness (g) & 45.79 & 35.12 & 17.51 & 15.32 \\
Work of shear (g.s) & 25.30 & 17.98 & 5.38 & 4.21 \\
Work of adhesion (g.s) & 41.19 & 34.79 & 13.38 & 12.57 \\
Stickiness (g) & 53.59 & 42.89 & 13.63 & 10.28
\end{tabular}

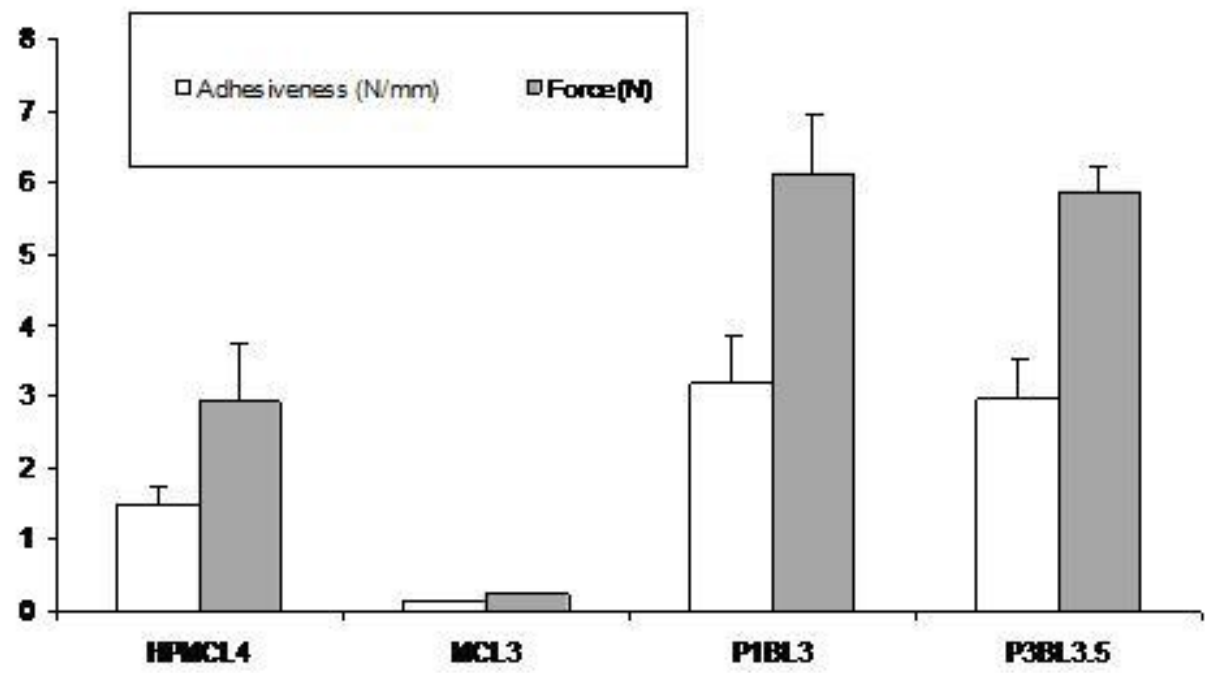

Fig. 5. Comparative bioadhesive strength of gels

In vitro permeation studies

In vitro release studies of various gels were carried out to estimate the amount of drug that is able to cross the biological membrane. A comparative in vitro permeation profile of DTZ through dialysis membrane from gel formulations is presented in Figure 6 . The data from the release profile was fitted to various models and coefficients of regressions have been enlisted in Table 4. 


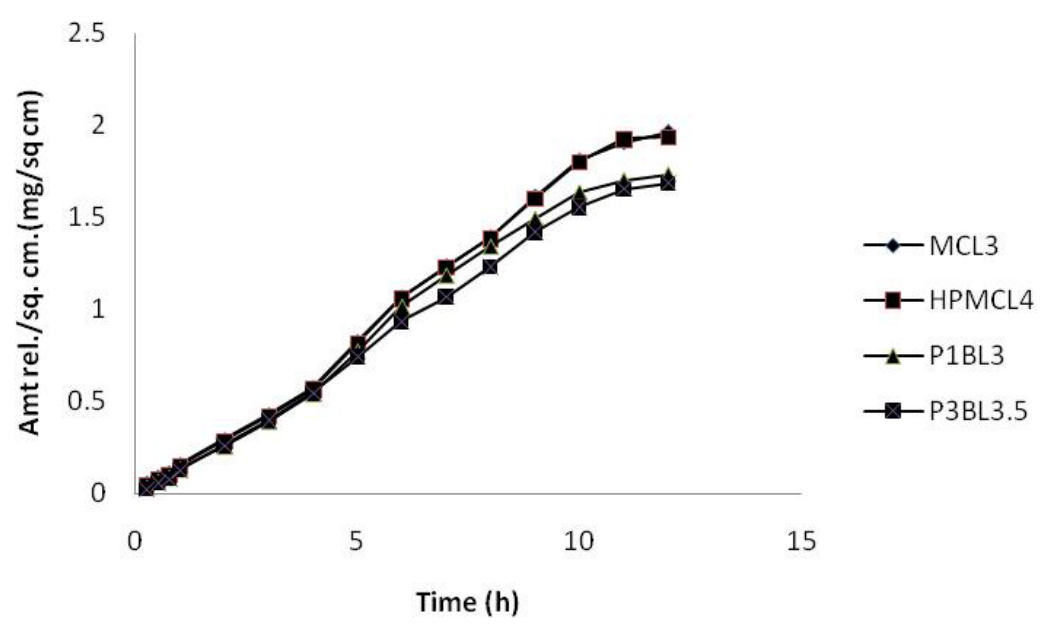

Fig. 6. Comparative in vitro permeation profile of DTZ through dialysis membrane from gel formulations

Tab. 4. The values of $r^{2}$ after fitting the release data to various models

\begin{tabular}{llccr}
\hline \multicolumn{1}{c}{ Model } & HPMCL4 & MCL3 & P1BL3 & P3BL3.5 \\
\hline Zero order & $r^{2}=0.9931$ & $r^{2}=0.9939$ & $r^{2}=0.9883$ & $r^{2}=0.9944$ \\
First order & $r^{2}=0.9931$ & $r^{2}=0.9939$ & $r^{2}=0.9884$ & $r^{2}=0.9945$ \\
Higuchi & $r^{2}=0.9168$ & $r^{2}=0.9172$ & $r^{2}=0.9271$ & $r^{2}=0.9308$ \\
Hixon-Crowell & $r^{2}=9931$ & $r^{2}=0.9939$ & $r^{2}=0.9884$ & $r^{2}=0.9945$ \\
Peppas & $r^{2}=0.9955$ & $r^{2}=0.9948$ & $r^{2}=0.9957$ & $r^{2}=0.9981$
\end{tabular}

\section{Stability testing}

Gel formulations were visually inspected for everyday for first week and then were inspected at intervals of 1, 2, 3 and 6 months respectively. All the four gel formulations did not show any appreciable change in gel clarity and color ratifying physical stability of prepared gel formulations. Further, no obnoxious odor was perceptible from any gel formulation. However, the consistency of PEO gels was altered. HPMCL4 and MCL3 gels were found to be stable at room temperature and at accelerated conditions $\left(40^{\circ} \mathrm{C} \pm 2^{\circ} \mathrm{C}\right.$, $75 \% \pm 5 \% \mathrm{RH}$ ). Even after exposure to heat and humidity, not much change was observed in $\mathrm{pH}$ of the gels. The rheograms and various power law equation parameters of these gels were found comparable at various time points in the accelerated stability study. All other parameters were also observed to be comparable. However, PEO gels failed in accelerated stability studies at one-month sample. These gels were then stored in refrigerator $\left(8\right.$ to $\left.15^{\circ} \mathrm{C}\right)$ and all the performance parameters were found to be comparable at each time point of the study. It could be concluded that PEO gels were unstable at room temperature but stable when stored in a refrigerator. Rheology of PEO gels was found to be not much altered after freeze thaw studies.

\section{Cloth staining}

This study is very important to ascertain the patient compliance of the gels. Patients generally resist rectal gels as they stain clothes. It was observed that all the four gels did 
not stain any of the fibre blends tested, even after prolonged exposure and accelerated drying. It could be concluded that none of the four gels stained any of the tested fibres.

\section{Clinical studies}

Twenty four consecutive patients (16 males) were included in the study. Four gel formulations (HPMCL4, MCL3, P1BL3, P3BL3.5) were given to six patients each. The baseline score for each patient at the time of recruitment (as evident from GIQLI) was recorded and the score of each patient was improved at the end of 8 weeks. No adverse effects were reported by any of the patients. The four gels were compared on the basis of GIQLI score and patient's compliance score. The study showed that $2 \%$ DTZ gel was effective in improving the quality of life of all the patients. There was a significant improvement of symptoms of pain and bleeding. The paired t-test using Sigmastat program (2.0) for windows was used for the comparison of score before and after treatment. If there was a significant improvement in quality of life following treatment, $a p$ value of less then 0.05 was considered significant. There was a significant improvement in quality of life of patients with HPMCL4 $(P<0.001), M C L 3(P<0.001), P 1 B L 3(P=0.001)$ and P3BL3.5 $(P<0.007)$. The difference of value between the scores before and after the application of each gel was compared using one-way ANOVA. No statistically significant difference was found among any of the gels $(P=0.931)$. The gels were also compared on the basis of patient compliance scores. Patient compliance scores were compared using one-way ANOVA followed by Dunnet's test. The difference in various gels was found to be statistically significant. The compliance scores of HPMCL4 were found to be greater then MCL3, P1BL3 and P3BL3.5 (P=0.007).

\section{Effect of DTZ gel in healthy volunteers}

DTZ causes a fall in blood pressure, therefore each formulation was applied by healthy volunteers $(n=6)$ to study the possible side effects of DTZ. As a control, blood pressure of each patient was measured at night and next morning for two consecutive days. The values of blood pressure before and after application were compared and no significant difference was observed in the blood pressure of the volunteers after application of the gels.

\section{Conclusions}

The method for analysis of DTZ was validated for accuracy, precision, reproducibility etc. Hydrogels of diltiazem hydrochloride with hydrophilic polymers viz., MC, HPMC and PEO were successfully prepared. The drug content of all the formulations was found to be within the limits. Various polymer concentrations for optimized formulations were found to $4 \%, 3 \%, 3.5 \%$ for HPMC, MC and PEO respectively. At these concentrations, gels could not be broken upto a shear stress of $500 \mathrm{~Pa}$. Beyond these concentrations, the formulations were of very high viscosity and adversely affected the characteristics required for a topical preparation. HPMC and MC gels were prepared in water while PEO gels were prepared in a buffer of $\mathrm{pH} 3.5$. When PEO gels were prepared in water, it led to the precipitation of DTZ in the gels. Hence gels with PEO were prepared using buffer as a solvent. The values of flow index for HPMCL4 was highest $(0.419)$ followed by MCL3 (0.380), P3BL 3.5 (0.357) and P1BL 3 (0.329). Sensory properties account for the gel behaviour during packaging, extrusion from the container, application at the site, and in situ behaviour after application to the target site These include measures such as consistency, 
firmness, cohesiveness (attractive forces within the formulation) and work of adhesion (attraction between the formulation and the substrate). All these properties were determined using texture profile analysis. It was observed that HPMCL4 had maximum firmness, work of shear and stickiness and work of adhesion whereas P3BL3.5 had least firmness, work of shear and stickiness. The values of mean AUC (adhesiveness) and the force required to break adhesive bonds were higher for P1BL 3 and lowest for HPMCL4. It could be concluded from the cloth staining studies that none of the four gels stained any tested fibre. It could be concluded from freeze thaw study that PEO gels were stable if they are stored in refrigerator. However, the gels can be kept at room temperature for short periods of time if taken out for application. When four selected gel formulations (HPMCL4, MCL3, P1BL3 and P3BL3.5) were given to six patients each, GIQLI score of each patient was improved at the end of 8 weeks. No adverse effects were reported by any of the patients.

\section{Authors' Statements}

\section{Competing Interests}

The authors declare no conflict of interest.

\section{Informed Consent \& Ethical Approvals}

The institutional and (inter)national ethical guides for experiments on human subjects were followed and informed consent was obtained. See the methods section for details.

\section{References}

[1] Kirsch J.

Anal fissure.

Wien Med Wochenschr. 2004; 154: 69-72.

doi:10.1007/s10354-004-0038-3

[2] Antropoli C, Perrotti P, Rubino M, Martino A, De Stefano G, Migliore G, Antropoli M, Piazza P. Nifedipine for local use in conservative treatment of anal fissures: preliminary results of a multicenter study.

Dis Colon Rectum. 1999; 42: 1011-1015.

doi:10.1007/BF02236693

[3] Corman M. Anal Fissure.

In: Corman $\mathrm{M}$, editor. Colon and rectal surgery.

Philadelphia, PA: Lippincott-Raven; 1998. p. 206-223.

[4] Dziki A, Trzcinski R, Langner E, Wronski W.

New approaches to the treatment of anal fissure.

Acta Chir lugosl. 2002; 49: 73-75.

doi:10.2298/ACI0202073D

[5] Acheson AG, Scholefield JH.

Pharmacological advancements in the treatment of chronic anal fissure.

Expert Opin Pharmacother. 2005; 6: 2475-2481.

doi:10.1517/14656566.6.14.2475

[6] Lubowski DZ.

Anal fissures.

Aust Fam Physician. 2000; 29: 839-844.

PMid:1108386 
[7] Allan A, May R.

Anal fissure.

Br J Hosp Med. 1985; 33: 41-43.

PMid:3971079

[8] Dhawan S, Chopra S.

Nonsurgical approaches for the treatment of anal fissures.

Am J Gastroenterol. 2007; 102: 1312-1321.

doi:10.1111/j.1572-0241.2007.01203.x

[9] Hager T.

[Anal fissure.]

Ther Umsch. 1997; 54: 190-192.

PMid:9221541

[10] Haq Z, Rahman M, Chowdhury RA, Baten MA, Khatun M.

Chemical sphincterotomy--first line of treatment for chronic anal fissure.

Mymensingh Med J. 2005; 14: 88-90.

PMid:15695964

[11] Gupta PJ.

Current treatment options for fissure-in-ano.

J Med Liban. 2004; 52: 33-38.

PMid: 15881700

[12] Nyam DC, Pemberton JH.

Long-term results of lateral internal sphincterotomy for chronic anal fissure with particular reference to incidence of fecal incontinence.

Dis Colon Rectum. 1999; 42: 1306-1310.

doi:10.1007/BF02234220

[13] Garcia-Aguilar J, Belmonte C, Wong WD, Lowry AC, Madoff RD.

Open vs. closed sphincterotomy for chronic anal fissure: long-term results.

Dis Colon Rectum. 1996; 39: 440-443.

doi:10.1007/BF02054061

[14] McCallion K, Gardiner KR.

Progress in the understanding and treatment of chronic anal fissure.

Postgrad Med J. 2001; 77: 753-758.

doi:10.1136/pmj.77.914.753

[15] Garrido R, Lagos N, Lattes K, Abedrapo M, Bocic G, Cuneo A, Chiong H, Jensen C, Azolas R, Henriquez A, Garcia C.

Gonyautoxin: new treatment for healing acute and chronic anal fissures.

Dis Colon Rectum. 2005; 48: 335-343

doi:10.1007/s10350-004-0893-4

[16] Attia MA, El-Gibaly I, Shaltout SE, Fetih GN.

Transbuccal permeation, anti-inflammatory activity and clinical efficacy of piroxicam formulated in different gels.

Int J Pharm. 2004; 276: 11-28.

doi:10.1016/j.ijpharm.2004.01.041

[17] Kelner A, Schacht EH.

Tailor-made polymers for local drug delivery: release of macromolecular model drugs from biodegradable hydrogels based on poly(ethylene oxide).

J Control Rel. 2005; 101: 13-20.

doi:10.1016/j.jconrel.2004.09.010 
[18] Jones DS, Woolfson AD, Brown AF, Coulter WA, McClelland C, Irwin CR.

Design, characterisation and preliminary clinical evaluation of a novel mucoadhesive topical formulation containing tetracycline for the treatment of periodontal disease.

J Control Rel. 2000; 67: 357-368.

doi:10.1016/S0168-3659(00)00231-5

[19] Franz TJ.

Percutaneous absorption on the relevance of in vitro data.

J Invest Dermatol. 1975; 64: 190-195.

PMid:123263

[20] USP-XXII. Rockwille, MD: United States Pharmacopoeial Convention Inc., Twinbrook Parkway.

[21] Buckley MM, Grant SM, Goa KL, McTavish D, Sorkin EM.

Diltiazem. A reappraisal of its pharmacological properties and therapeutic use.

Drugs. 1990; 39: 757-806.

PMid:2191851

[22] Jones DS, Woolfson AD, Djokic J, Coulter WA.

Development and mechanical characterization of bioadhesive semi-solid, polymeric systems containing tetracycline for the treatment of periodontal diseases.

Pharm Res. 1996; 13: 1734-1738.

doi:10.1023/A:1016413428473 